\title{
Análisis de la eficacia del uso de la terapia de juego y la parentalidad positiva en la atención temprana de niños con trastorno del espectro autista (TEA)
}

\section{Analysis of the effectiveness of the use of play therapy and positive parenting in the early care of children with autism spectrum disorder (ASD)}

DOI: $10.46932 / \mathrm{sfjdv2n5-017}$

Received in: Jun 1st, 2021

Accepted in: Sep 30th, 2021

\author{
Alba Rodríguez Capdepón \\ Maestra de Educación Infantil. Universidad de Murcia \\ Ángela Pérez Lencina \\ Maestra de Educación Infantil. Universidad de Murcia \\ María Moreno Ruiz \\ Maestra de Educación Infantil. Universidad de Murcia
}

\section{RESUMEN}

En los últimos años, el número de personas con Trastorno del Espectro Autista (TEA) ha aumentado. De ahí, la importancia de un diagnóstico precoz, así como una intervención temprana. Existen varias metodologías utilizadas en la actualidad a la hora de tratar a los niños con este diagnóstico. Dada la novedad que presenta la parentalidad positiva y el uso de juegos en la terapia de niños con TEA, hemos considerado interesante comprobar la efectividad y puesta en práctica de dichos tratamientos. Por ello, el presente artículo se basa en una revisión sistemática de varios artículos relacionados con la terapia parental y filial, así como la terapia de juego en el tratamiento de niños con Trastorno del Espectro Autista (TEA). Para ello, se ha realizado una búsqueda con cuatro palabras clave en cinco bases de datos distintas. Nuestro objetivo principal es comprobar si dichas terapias son realmente efectivas, así como su uso en la actualidad. Para ello, se ha realizado un resumen de los artículos seleccionados, discutiendo los resultados obtenidos en cada uno de ellos. De este modo, hemos podido comprobar si nuestros objetivos se cumplían, llegando a la conclusión de que tanto la terapia parental como la terapia de juego son beneficiosas en los niños con TEA, si bien se precisa de una mayor investigación de su puesta en práctica.

Palabras clave: TEA, parentalidad positiva, terapia de juego, revisión sistemática e intervención temprana.

\footnotetext{
ABSTRACT

In recent years, the number of people suffering from Autism Spectrum Disorder (ASD) has increased. Hence, the importance of an early diagnosis as well as an early intervention. There are several methodologies currently used when treating children with this diagnosis. Given the novelty of the use of positive parenting and play therapies of children with ASD, we have considered it interesting to verify the effectiveness and implementation of these treatments. Therefore, this article is based on a systematic review of several articles related to parental and filial therapy, as well as play therapy in treatment with children suffering from ASD. To that aim, a research using four key words has been carried out in five distinct databases. Our main aim is to see if said therapies are really effective, and if they are used in present therapies. For this reason, a summary of the selected articles has been made, discussing the results obtained in each one of them. In that way, we were able to verify whether our objectives were met,
} 
reaching the conclusion that both parental and play therapies are beneficial for children with ASD, although a further investigation for its implementation is required.

Key words: ASD, positive parenting, play therapy, systematic review and early intervention.

\section{INTRODUCCIÓN}

El Trastorno de Espectro Autista, conocido como TEA, es un trastorno del neurodesarrollo que afecta a las personas en la vida diaria. De hecho, y de acuerdo con la Organización Mundial de la Salud, aproximadamente un $1 \%$ de la población mundial se ve afectada por dicha enfermedad, y cada día son más el número de niños que son diagnosticados con dicho trastorno. Es por ello que su detección temprana es de vital importancia, para poder intervenir y tratar a los niños desde una edad temprana. Hoy día, existen diferentes tratamientos que se llevan a cabo con dichos niños para estimular y desarrollar las áreas sociales y comunicativas afectadas. Algunas de estas terapias, y las más novedosas, son aquellas relacionadas con la parentalidad positiva, así como las terapias de juego, muchas de las cuales están relacionadas con el uso de las nuevas tecnologías. Por este motivo, en este artículo se va a llevar a cabo una revisión sistemática en el que se compararán aquellos estudios relacionados con dichas terapias para comprobar su efectividad y su puesta en práctica.

\section{OBJETIVOS}

El obetivo principal de este artículo es comprobar si, desde el punto de vista de la atención temprana, se produce una mejora en los niños con Trastorno del Espectro Autista utilizando técnicas alternativas a las convencionales como la terapia de juego y la parentalidad positiva. Con dicho fin, se efectuará una revisión sistemática.

Los objetivos específicos son los siguientes:

- Comprobar si la intervención de los padres influye de manera positiva en los niños con TEA.

- Valorar cómo afecta la terapia de juego a los niños con Trastorno del Espectro Autista.

- Verificar si dichas técnicas se ponen en práctica en la actualidad con los niños con TEA.

\section{METODOLOGÍA}

Se efectuó una búsqueda de las publicaciones relacionadas con nuestros objetivos en cinco bases de datos diferentes, ERIC, Psycharticles, Pubmed, Scopus y Web of Science. La búsqueda se realizó desde 
de enero a mayo de 2021, accediendo a todas las bases de datos a través del Escritorio virtual de la Universidad de Murcia (EVA).

\subsection{ESTRATEGIA DE BÚSQUEDA}

La estrategia de búsqueda utilizada, y los términos empleados fueron "ASD", "early intervention", "positive parenting", "play therapy". Por otro lado, los conectores seleccionados fueron "and" para buscar registros que incluyen todos los términos separados por el operador y "or" para buscar registros que incluyan cualquiera de los términos separados por el operador. Asimismo, estos términos pueden aparecer en cualquier lugar del documento. La búsqueda finalizó en mayo de 2021.

\subsection{CRITERIOS DE INCLUSIÓN}

Para este análisis los criterios de inclusión que han ayudado a hacer más fácil esta búsqueda han sido:

Criterio 1: período de publicación comprendido entre los años 2010 al 2020.

Criterio 2: documentos que ofrecen resultados empíricos de investigación.

Criterio 3: acceso al texto completo.

Criterio 4: estudios escritos en lengua inglesa o española.

Criterio 5: artículos relacionados con niños autistas de edad de 0 a 14 años.

\subsection{CRITERIOS DE EXCLUSIÓN}

En cuanto a los criterios de exclusión utilizados en este análisis son:

Criterio 1: artículos que fuesen tesis doctorales, estudios de revisión teórica, revisiones sistemáticas o meta-análisis que no fuesen en inglés o español.

Criterio 2: documentos que no pertenecían al rango de edad de la primera infancia (0-5 años) o edad escolar (5-14 años), obviando todos los estudios que sobrepasasen ese intervalo de edad.

Criterio 3: Trabajos que no se centran en el objetivo de este artículo si no que de una forma u otra, se desvirtúan dando pie a aspectos más generales que específicos, que es lo que se busca en esta revisión sistemática.

Una vez concluida la búsqueda de todos los documentos/artículos de esta revisión sistemática se obtuvo un total de 232 referencias. En el esquema se presenta como ha sido realizada la revisión de los artículos y la toma de decisiones para incluirlo en este artículo. "Web of Science" es un buscador amplio donde se recoge todo tipo de información. De este hemos seleccionado un total de 2 artículos y hemos descartado 185 que no se ajustaban a los criterios de inclusión de nuestra investigación debido a que eran 
artículos que no se centraban en el objetivo de nuestro artículo. Además, cuatro de estos artículos estaban duplicados en otra base de datos. En la base de datos "Pubmed", hemos seleccionado inicialmente un total de 26 artículos de los cuales dos estaban duplicados en otras bases de datos y 21 no cumplían con alguno de los criterios de inclusión, siendo 2 de ellos seleccionados para esta revisión. En la base de datos "Scopus" la búsqueda ha dado lugar a la selección de 2 artículos de los 6 que quedaban reflejados según los descriptores marcados. En la base "Pyscharticles" hemos obtenido un total de 3 artículos de los cuales no hemos podido utilizar ninguno ya que el rango de edad era superior al establecido en los criterios de inclusión. Por último, en la base "ERIC" hemos encontrado un total de 9 artículos de los cuales ninguno ha sido útil para esta tarea, pues ninguno de estos se centraba en el tema objeto de estudio. Asimismo, resaltar que no se encontró ningún documento que relacionará el término "positive parenting” con el resto de términos mencionados. En resumen, se encontraron un total de 6 artículos que se adaptan a los criterios preestablecidos.

Con la revisión de los abstracts se ha podido descartar más del 90\% de las búsquedas reflejadas en las referencias. La mayoría de los documentos han sido descartados debido a que no se ajustaban al objetivo principal del artículo ya que, muchos de estos no se centraban en tratamiento de patologías asociadas al Trastorno del Espectro Autista. Desgraciadamente, muchos de estos estudios estaban orientados a alumnos y alumnas de otros niveles educativos. Como bien ha quedado reflejado, 19 de estos han sido revisados a texto completo de los cuales 6 de ellos van a ser utilizados para contrastar resultados y llegar a conclusiones lo más certeras posibles. Para finalizar se ha observado que, tras una revisión profunda pero a la vez concisa, se ha seleccionado un total de 6 artículos los cuales cumplen en la medida de lo posible el objetivo principal de esta revisión sistemática.

\section{RESULTADOS}

Una vez aplicados los criterios de inclusión y exclusión hemos seleccionado seis artículos de carácter científico, los cuales recogen estudios acerca de diferentes métodos y estrategias relacionadas con la familia y la terapia de juego para ayudar a los niños con autismo. A continuación, podemos observar los diferentes resúmenes de estos estudios, así como una tabla resumen de recogida de datos en el que se presentan: el nombre de los diferentes artículos utilizados, los autores de cada investigación, el objetivo, la muestra, los instrumentos utilizados para el desarrollo del estudio, los resultados y las conclusiones.

“The Effect of Filial Therapy on the Parenting Stress of Mothers of Children with Autism Spectrum Disorder", llevado a cabo por Kiyani1, Mirzai1, Hosseini, Sourtiji, Hosseinzadeh, Ebrahimi (2020). 
En esta investigación se expone que diversos estudios han demostrado que las madres de niños con TEA (Trastorno del Espectro Autista) están sometidas a más estrés que las madres con niños que no poseen este trastorno. Además, se destaca que el estado de ánimo de la madre afecta a los problemas de conducta del TEA, y esto puede perturbar el proceso de recuperación de estos niños. Por tanto, resulta necesario encontrar una manera de reducir el estrés de las madres con niños con TEA y con esto aumentar el rendimiento de los niños.

En este artículo se afirma que el mejor método de intervención, que involucre tanto a la madre como a su hijo con TEA, es la terapia de juego. La terapia filial es una terapia lúdica centrada en la familia que utiliza el juego como herramienta primaria para ayudar a los padres y a los niños a comunicarse, trabajar en sus problemas, y construir una relación. Fue introducido por Bernard y LouiseGuerney. El modelo de terapia filial de diez sesiones desarrollado por Gary Landreth es uno de los tratamientos más utilizados, el cual se denomina Terapia de Relación Padre-Hijo. El objetivo de este estudio era investigar el efecto que tiene la terapia filial en la reducción del estrés de madres de niños con Trastorno del Espectro Autista.

Se trata de un estudio experimental con diseño pre test y post test con un grupo control. La muestra del estudio consta de aquellas madres que tienen hijos con TEA de entre 4 y 12 años y están remitidos a los centros de terapia ocupacional y autismo de la ciudad de Isfahán, Irán. El total de participantes fue de 32, 9 niñas (5 en el grupo de intervención y 4 en el grupo de control) y 23 niños (11 en el grupo de intervención y 12 en el grupo de control). Los sujetos que conformaron ambos grupos no mostraban diferencias significativas entre ellos.

Las madres de estos niños tuvieron que completar un formulario demográfico, denominado GilliamAutismEscala de Clasificación-2 (GARS-2), el cual se trata de una lista de verificación diseñada por Gilliam (1994) para diagnosticar autismo en el grupo de edad de 3 a 22 años. Asimismo, también completaron el Formulario breve de estrés de Abidin (PSI-SF). Dicho formulario tiene 36 ítems desarrollados por Abidin y tres subescalas: Estrés Parental, Interacción Disfuncional Padre-Niño, y Dificultad en la conducta del hijo.

Los participantes fueron divididos de forma aleatoria en los dos grupos de intervención y control. El grupo de intervención recibió 10 sesiones de terapia filial una vez a la semana, de dos horas cada sesión. Por otro lado, el grupo de control continuó con el tratamiento rutinario.

Tras la intervención, las madres completaron de nuevo el test de Índice de Estrés Parental. La información recolectada fue analizada utilizando ANCOVA, una prueba T para muestras relacionadas y una prueba $\mathrm{T}$ para muestras independientes. Por otro lado, el test Shapiro-Wilk fue utilizado para 
determinar la normalidad de la distribución datos del test de Índice de Estrés Parental (PSI-SF) en su fase pre-test y post-test.

En cuanto a los resultados obtenidos, los resultados de la Prueba T para muestras relacionadas no mostraron diferencias estadísticamente significativas entre los dos grupos en la fase pre-test $(\mathrm{P}=0,679)$, pero si una diferencia significativa en la fase Post-test $(\mathrm{P}=0,010)$. Sin embargo, los resultados de la Prueba $\mathrm{T}$ para muestras independientes mostraron la diferencia en las puntuaciones generales entre Pre-test y post-test del estrés parental en el grupo de control no fueron significativas $(\mathrm{P}=0,268)$ pero había una diferencia significativa en el grupo de intervención $(\mathrm{p}=0,001)$. Los resultados de ANCOVA mostraron que la puntuación Pre-test del estrés parental tuvo un efecto en el resultado general después de la intervención, por lo que podría explicar el $82 \%$ de la variación total del estrés parental. La puntuación de estrés fue significativamente diferente entre los dos grupos $(\mathrm{p}=0,001)$. De acuerdo con los resultados, la terapia filial de grupo puede explicar el $54 \%$ de los cambios en el estrés parental total de las madres.

Como conclusión, este estudio afirma que el autismo es un trastorno muy complejo, que incluso en algunas ocasiones es difícil para personas especializadas como los psiquiatras. Además, los miembros de las familias que tienen hijos con este trastorno piensan a menudo que sus hijos y su desarrollo es la primera prioridad y a veces tienden a olvidarse de sí mismos y de su salud mental e incluso física. En lo que concierne a la Terapia de relación padre-hijo, manifiesta que es una técnica sin limitaciones que puede ayudar tanto a la madre como al hijo en su tratamiento. Asimismo, puede reducir el estrés y la ansiedad de las madres creando una mayor aceptación hacia sus hijos de manera positiva en el proceso terapéutico y de desarrollo, tanto de manera directa como indirecta. Por lo que se puede concluir que la terapia filial de grupo puede ayudar a una mayor aceptación de dichos niños por sus padres, a la vez que reduce el estrés parental mediante la mejora de la relación padre-hijo.

"Effects of a Brief Early Start Denver Model (ESDM) - Based Parent Intervention on Toddlers at Risk for Autism Spectrum Disorders: A Randomized Controlled Trial". Rogers, Estes, Lord, Vismara, Winter, Fitzpatrick, Guo y Dawson. (2012)

El objetivo principal de este estudio es examinar la eficacia de un tratamiento de baja intensidad, exactamente de 12 semanas, 1 hora por semana de contacto con el terapeuta, a través de varias intervenciones con padres de niños pequeños con patrones de autismo de 12 a 24 meses.

Se llevó a cabo un ensayo controlado de forma aleatoria que involucró a 98 niños y familias y que se llevó a cabo en tres lugares distintos: Sacramento, Seattle y Ann Arbor. De estos 98 niños, 24 de ellos tenían un hermano con TEA, además 26 niños tenían hermanos con otros trastornos del desarrollo, incluyendo retrasos del habla, problemas de aprendizaje, Síndrome de Down o discapacidades intelectuales entre otros. 
En cuanto a la metodología utilizada se llevaron a cabo diferentes recursos: en primer lugar, se usaron cuestionarios con las familias. El primero de ellos es Early Screening of Autistic Traits Questionnaire (ESAT), una entrevista con los padres de niños de 12 a 15 meses. El segundo de ellos es Infant Toddler Checklist (ITC), un cuestionario parental para determinar los desórdenes en la comunicación, el cual se utilizó con todos los niños de este estudio. Finalmente, el tercer cuestionario es el denominado Modified Checklist forAutism in Toddlers (M-CHAT), un cuestionario parental de 23 ítems desarrollado para observar síntomas de autismo. Esta herramienta fue utilizada en niños de 16 a 24 meses.

En cuanto a aquellas medidas que se utilizaron para el diagnóstico del autismo, se utilizó el Autism Diagnostic Observation Scale for Toddlers (ADOS-T), el cual se trata de una evaluación observacional que mide los comportamientos sociales y comunicativos, así como comportamientos repetitivos diagnósticos de autismo.

En tercer lugar, se llevaron a cabo tres métodos distintos para evaluar el desarrollo de los niños. El primero de ellos fue Mullen Scales of Early Learning (MSEL), el cual se trata de una evaluación estandarizada que se llevó a cabo al comienzo del tratamiento y 12 semanas después. Para ello se creó tres puntuaciones de cociente de desarrollo: un cociente de desarrollo verbal, uno no verbal y un cociente de desarrollo general creado a partir de estas subescalas. Por otro lado, se utilizó MacArthur-Bates Communicative Development Inventory: Words and Gestures (MCDI). Este recurso es un listado de vocabulario de 396 palabras para recopilar todas aquellas palabras, gestos y vocabulario receptivo que el niño ha mostrado en las pasadas semanas. Finalmente, se ha utilizado Vineland Adaptive Behaviour Scales, Second Edition (VABS II). Esta escala examina cuatro áreas de comportamiento adaptativo: comunicación, cuidado personal, habilidades sociales y habilidades motoras. Se llevó a cabo por teléfono al inicio y al final de la intervención.

Un cuarto punto en el estudio fue Child Moderating Variables, que incluye la imitación, así como, la orientación a estímulos sociales, no sociales y atención conjunta. En lo que respecta a la imitación, se llevaron a cabo doce tareas de imitación mediante un sondeo de juego de unos diez minutos aproximadamente, que pretendía analizar imitaciones de objetos familiares y nuevos, así como imitaciones de gestos manuales y faciales. Por otro lado, una breve tarea desarrollada por Dawson et al. (2010) se llevó a cabo para evaluar la implicación social del niño. Dicha tarea incluía tres tipos de sondeos: cuatro ruidos "sociales", cuatro ruidos "no sociales" y cuatro invitaciones a la interacción. El investigador graba si el niño gira la cabeza o desvía la mirada hacia el sonido, durante al menos 10 segundos.

Finalmente, se llevaron a cabo medidas parentales. La primera de ellas, se trata de un cuestionario demográfico que contiene preguntas relativas a la composición familiar, tamaño de la familia, el número de residentes en el hogar, el nivel educativo de los padres, la ocupación de los padres y su ingreso anual. 
Asimismo, se utilizó el Symptom Checklist-90-R (SCL-90-R) (Listado de síntomas 90R), un cuestionario de 15 minutos que consta de 90 ítems, el cual fue utilizado para recolectar información sobre las dificultades de salud mental de los padres. Otra de estas medidas, se trata de ESDM Parent Fidelity Tool. La herramienta de fidelidad P-ESDM es un sistema de calificación de cinco puntos basado en la escala Likert de 13 comportamientos de los padres. Las interacciones de juego entre padres e hijos con un conjunto específico de juguetes y la instrucción de "jugar como lo hace normalmente en casa", se grabaron en video en ambos puntos de la evaluación. El cuarto recurso utilizado ha sido Working Alliance Scale for Interventions with Children. Este recurso fue utilizado para examinar las respuestas de las familias a la intervención temprana del terapeuta que trabajó con ellos al final de las 12 semanas. Finalmente, se tuvo en cuenta el denominado Child Intervention Hours, es decir, todas las intervenciones de todo tipo fueron examinadas dos veces: la primera de ellas, T1, que incluyó todas las horas recibidas hasta ese momento, y la segunda, T2, que incluyó el número promedio de horas recibidas por semana entre T1 y $\mathrm{T} 2$.

El tratamiento P-ESDM (Modelo de Intervención Temprana de padres), consiste en doce sesiones consecutivas cada una de una hora. Estas sesiones de intervención se llevaron a cabo en las tres clínicas universitarias previamente mencionadas por terapeutas altamente experimentados. La primera sesión se centraba en desarrollar los objetivos de aprendizaje de los niños y las dos últimas sesiones fueron sesiones de mantenimiento, así como una revisión del progreso. De la sesión número 2 a la 10, los padres fueron entrenados en diez técnicas de intervención del modelo ESDM. Las sesiones comenzaban con un breve periodo de interacción de 5 minutos entre el padre y el niño. A continuación, los terapeutas introducían una temática nueva cada semana a través de una descripción verbal y materiales escritos. Después, el padre ponía en práctica la técnica de juego indicada hasta que éste demostraba su implementación en un $80 \%$ o mayor. Cada sesión concluía con un debate entre el terapeuta y el padre sobre esta nueva habilidad y sus diferentes formas de implementación.

En cuanto a los resultados obtenidos tras el estudio, se observó que la asignación de grupo no tiene ningún efecto sobre las características de la interacción entre padres e hijos o el resultado de los niños. Ambos grupos de padres mejoraron sus habilidades de interacción y ambos grupos de niños progresaron. Los padres que recibieron P-ESDM demostraron alianzas de trabajo significativamente más fuertes con sus terapeutas que el grupo de control. Los niños del grupo de control recibieron mucho más tiempo de intervención que los niños del grupo P-ESDM. En lo que respecta al grupo en general, los factores de que el niño fuera más pequeño y el mayor número de horas de intervención fueron positivos, relacionados con una mejora del comportamiento de los niños en la mayoría de las variables estudiadas. 
Como conclusión, podemos decir que el principal problema de este estudio fue su diseño. Uno de los motivos fue que el grupo de control recibió un mayor número de horas de intervención con respecto al grupo que llevó a cabo el modelo P-ESDM. Además, es bastante probable que los servicios que recibió el grupo de control incluyera el entrenamiento de los padres, y por tanto haya sido similar al grupo PESDM. Una tercera debilidad en el diseño del estudio puede deberse al calendario establecido, ya que no recibían una intervención completa hasta el final de dicho periodo, por lo que no ha habido tiempo para que los cambios en los comportamientos de los padres hayan podido afectar al comportamiento del niño. Por otro lado, se considera que otro problema derivado de este estudio sea la división de los grupos de forma aleatoria.

\section{“Using Augmented Reality to Elicit Pretend Play for Children with Autism" Bai, Blackwell}

\section{y Coulouris (2015)}

El objetivo de este estudio es desarrollar un sistema interactivo que explora el potencial de la Tecnología de Realidad Aumentada para conceptualizar visualmente la representación de la simulación dentro de un entorno de juego abierto, el cual es comparado con una situación no asistida por ordenador.

El sistema de Realidad Aumentada (RA) ha sido diseñado bajo la representación de vista de espejo, donde la realidad es enriquecida mediante dicha realidad aumentada. Se eligió la representación de espejo porque permite a los usuarios interactuar con el sistema sin necesidad de llevar o sostener el equipamiento y así poder manipular juguetes físicos con ambas manos.

Asimismo, se escogió como temática un juego de coches, trenes y aviones, ya que los niños autistas a menudo muestran un interés obsesivo por las máquinas. Para aumentar la unión con el sistema, se añadieron estímulos visuales adicionales a los vehículos, como manivelas y ruedas giratorias.

Dicha realidad aumentada se ha implementado mediante el uso de marcadores, ya que nos permitía una mayor flexibilidad de elección del objeto. El experimento consiste en dos modalidades: con y sin RA. En cada modalidad se llevan a cabo tres tareas y su orden es al azar. Las hipótesis de dicho experimento son: Hipótesis A: no hay diferencias significativas en la frecuencia de juego simulado entre el uso de realidad aumentada y la modalidad sin ordenador. Por otro lado, la Hipótesis B infiere que no hay diferencias significativas en la duración del juego simulado entre el uso de RA y la modalidad sin su uso.

Los participantes de este estudio fueron doce niños diagnosticados con Trastorno del Espectro Autista de entre 4 y 7 años: 10 niños y 2 niñas. Antes de llevar a cabo el experimento, se visitó a los participantes en su casa para recolectar información sobre su autismo y condiciones del lenguaje. Para ello se utilizó la Escala de Autismo en la Infancia basada en entrevistas con los padres así como observación directa. También se evaluó su edad mental. En lo que respecta a su uso del lenguaje usando la Escala de Vocabulario de Inglés con Imágenes (British Picture Vocabulary Scale). 
Tras dicha entrevista, se observó que el nivel del uso de objetos en el juego entre los participantes, era mediana y moderadamente inapropiada. En cuanto a la frecuencia de juego de simulación, era la siguiente: 3 participantes lo hacían frecuentemente, 4 participantes algunas veces, 4 participantes rara vez, y 1 participante nunca lo hacía. Añadir que todos los participantes están familiarizados con el uso de ordenadores, usándolo la mayoría de forma diaria.

Los procedimientos del experimento eran consistentes, tanto en la modalidad de RA como la configuración sin ordenador: en primer lugar, se realiza una breve introducción para recordar a los participantes que deben jugar dentro del área indicada, y que pueden jugar con lo que quieran de la mesa, parando después de 5 minutos. Después, se inicia el juego, donde el investigador sostiene uno de los bloques y pregunta: “enséñame cómo puedes jugar con este bloque como si fuese un coche/tren/avión”. Durante la tarea, el investigador no debe dar ideas sobre juego simulado, si el niño se distrae, el investigador puede animarlo a continuar jugando y a usar los objetos físicos, sin embargo tan sólo lo hará en 3 ocasiones. Una vez pasados 5 minutos, el investigador esperará a que el niño termine con el juego y le dirá: "Muy bien. Vamos a parar y ponerlo todo en su sitio". Esto mismo se repetirá en cada una de las tareas, preguntando al participante para que nos dé información al finalizar cada modalidad.

En lo que respecta a los resultados de este experimento, se analizó la frecuencia del juego, su duración y el compromiso de los niños con el mismo. En lo que respecta a la frecuencia del juego, se analizó mediante el test Shapiro-Wilk. Tal y como se puede observar, la frecuencia de juego simulado es mayor en la modalidad de Realidad Aumentada, mientras que la frecuencia de juego constructivo era más alta en la modalidad sin ordenador. Se llevó a cabo una evaluación una prueba t para muestras dependientes y se demostró que existe una diferencia significativa entre el juego simulado (t $(9)=4.66, \mathrm{p}$ $<0.01)$ y el juego constructivo $(\mathrm{t}(9)=-4.91, \mathrm{p}<0.01)$.

Por otro lado, en lo que respecta a la duración del juego, el porcentaje de tiempo que los participantes usan en el juego simulado es significativamente mayor en la modalidad de realidad aumentada $(\mathrm{t}(9)=3.25, \mathrm{p}<0.01)$.

Finalmente, en lo que se refiere a la atención y cooperación de los niños, se ha evaluado su sonrisa de felicidad durante el juego mediante el test no paramétrico de Wilcoxon. También se ha utilizado como instrumento de evaluación un cuestionario que han completado los padres, cuyos datos están en concordancia con el compromiso de los niños en el juego.

Por tanto, como conclusión, podemos decir que los resultados indican un efecto positivo del aumento de la frecuencia del juego de simulación, su duración y relevancia con el sistema de RA en comparación con una configuración sin ordenador. Los participantes se mostraron muy comprometidos con el sistema de RA y produjeron una amplia gama de ideas de juego. Las diferencias individuales entre 
los participantes predicen una eficacia gradual para las diferentes condiciones autistas. El sistema de Realidad Aumentada tiende a tener el efecto más positivo en los niños que tienen el mayor retraso en el desarrollo, en el juego de simulación, y para los niños que realizan juegos de simulación en casa, el sistema de RA puede no proporcionar una mejora adicional.

\section{"Cognitive rehabilitation system for children with autism spectrum disorder using serious} games: A pilot study" Aresti-Bartolome y Garcia-Zapirain (2015)

Gracias a las TIC las personas con TEA pueden practicar sus fortalezas y trabajar sus debilidades, ayudándoles a aumentar su vocabulario y su comunicación.

El objetivo principal de este estudio piloto es evaluar objetivamente la interacción entre los niños con TEA y el terapeuta mediante juegos y técnicas de exploración. Para lograr este objetivo principal, se han establecido tres metas: la primera, desarrollar un sistema de juego que permita la rehabilitación de las áreas afectadas por TEA. La segunda, evaluar la interacción con las personas con TEA en el curso de las actividades incorporadas en el sistema mediante variables objetivas. Finalmente nuevo tercer objetivo es establecer indicadores objetivos que permitan comparar el comportamiento cognitivo entre personas con TEA y personas con un desarrollo normotípico.

En cuanto a la metodología utilizada, para analizar los datos se utilizó el programa SPSS. Asimismo, para establecer la significación entre el grupo control y el grupo clínico se empleó el test paramétrico de Mann-Whithney. De este modo, se pudo determinar si el sistema podía utilizarse como herramienta de rehabilitación. Por último, se utilizó la prueba del estadístico de Spearman para analizar la correlación entre el tiempo de respuesta mirando a los ojos del responsable de la sesión y el número de veces que esta interacción se realizó.

Los participantes del estudio fueron 20 niños de entre 3 y 8 años de edad que conformaban el grupo clínico. Estos niños habían sido diagnosticados con TEA y asistían regularmente a sesiones de tratamiento con profesionales. Asimismo, el grupo control estaba conformado por 20 niños con desarrollo normotípico, de edades comprendidas entre 3 y 8 años igualmente y sin ningún impedimento físico o mental. Los dos grupos fueron emparejados según sexo y edad.

Para alcanzar los objetivos establecidos en el estudio se han considerado tres aspectos para llevar a cabo el experimento de manera correcta: la configuración de los juegos, la selección del ambiente en el que se lleva a cabo el experimento y la óptima configuración de un rastreador ocular.

En lo que respecta a la configuración de los juegos, los niveles de dificultad y el modo de juego fueron definidos y establecidos como resultado de algunas sesiones que se llevaron a cabo con profesionales. Este sistema se centra en la rehabilitación cognitiva mediante la interacción entre el niño y el conductor de la sesión. Este sistema contiene un conjunto de juegos divididos en distintos niveles de 
dificultad (nivel 1, nivel 2 y nivel 3) y que implican recolectar elementos que aparecen en la pantalla. La dificultad de los niveles se definió en función del grado de interacción requerido y de la zona que se trabajaba en cada uno de ellos. Además del propósito del juego en sí, la finalidad es trabajar y evaluar la interacción con los responsables de las sesiones y el tiempo que les ha llevado interactuar. Es por ello que el juego para cada 30 segundos automáticamente o al cometer errores para forzar la interacción con el responsable de la sesión y evaluar sus habilidades comunicativas.

Por otro lado, en lo que respecta a la selección del entorno en el que se va a llevar a cabo el experimento, la clase estaba equipada con sillas y mesas adaptadas al niño, lo que contribuía a un entorno más cómodo para la realización de los juegos. Asimismo, dichas aulas no tenían ventanas, utilizando luz artificial, para que no se viese afectada la información recogida por el rastreador ocular.

Por último, se tuvo en cuenta la configuración y análisis del rastreador ocular. Éste se colocó en la parte inferior de la pantalla para obtener los datos de manera fiable. El niño debía sentarse sobre una silla alineada con el centro de la pantalla y colocada a 56 centímetros de ésta última.

En cuanto a los resultados obtenidos, se ha tenido en cuenta: los resultados de la interacción con el responsable de la sesión, los resultados del juego, así como los resultados del rastreador ocular.

Observando los resultados de la interacción de los participantes y el responsable de la sesión encontramos que el test de Mann-Whithney mostró que el tiempo de respuesta para el grupo clínico (M $=21.008, \mathrm{DE}=25,24)$ y el grupo de control evidenció diferencias significativas, debido a que el tiempo de reacción del primer grupo fue mayor que el segundo grupo. Además, el test indicó que había diferencias en relación al tipo de interacción de los niños participantes, ya que el grupo de intervención miró menos a los ojos del responsable de la sesión que el grupo de control. Asimismo, el grupo clínico necesitó más tiempo para interactuar al mirar a los ojos que el grupo de control. Sin embargo, para el grupo de control, el tiempo de reacción disminuyó independientemente del tipo de interacción. Por otro lado, el análisis descriptivo mostró que el grupo clínico interactuó por un período más largo mirando a los responsables de la sesión a los ojos, que a través de gestos o palabras.

Para evaluar la interacción con el juego se analizaron tres aspectos: el porcentaje de personas que no superaron ningún nivel, los aciertos y los errores durante el juego y la posición del puntero. Así, se ha podido observar que el 66\% de los niños del grupo clínico que no pasaron el nivel 3 interactuaron menos durante dicho nivel. Además, la prueba estadística de Mann-Whithney indicó que la única diferencia significativa entre ambos grupos se dio en los aciertos y errores; sin embargo la diferencias de posición del puntero no fueron significativas.

Por último, en cuanto a los resultados obtenidos con el rastreador ocular, se confirma que no había diferencias significativas entre las posiciones medias de las miradas cuando los participantes estaban 
jugando. Sin embargo, sí se encontraron diferencias durante las pausas del juego: el grupo de control mantenía sus ojos en la parte de arriba a la derecha de la pantalla, mientras que el grupo clínico lo hacía hacia la parte de abajo a la derecha.

Como conclusión, este estudio muestra que existen limitaciones, ya que los participantes solo trabajan con la aplicación, faltando así la interacción con alguien más, permitiendo trabajar las habilidades sociales. Esta primera fase de estudio nos ha ayudado a obtener resultados preliminares que se usarán como indicadores de evaluación para ambos grupos. Así, un estudio longitudinal nos permitirá verificar cómo evolucionan estos parámetros y si el grupo clínico se acerca a los resultados obtenidos por el grupo de control. Por lo tanto, los autores concluyen que las nuevas tecnologías hacen posible, no sólo crear ambientes adaptados a las necesidades de cada persona, sino también usarlos como herramienta de rehabilitación cognitiva.

"Brief Report: Longitudinal Improvements in the Quality of Joint Attention in Preschool Children with Autism". Lawton y Kasari (2011)

El estudio tenía dos objetivos. Primero, saber si la frecuencia de afecto positivo compartido utilizado durante intervenciones de atención conjunta aumentó más para los niños que habían sido asignados al azar a una intervención de atención conjunta o una intervención de juego simbólico que un grupo de control. Segundo, saber si la frecuencia del afecto positivo compartido mediante expresiones durante la atención conjunta aumentó más en estos grupos de tratamiento que en el grupo de control.

Los resultados principales incluían la cantidad de atención conjunta, la duración del juego, duración del compromiso conjunto así como el lenguaje expresivo. Para ello 52 niños en edad preescolar con autismo fueron asignados al azar a una intervención de atención conjunta, una intervención de juego simbólico o un grupo de control.

Se administró una amplia batería de evaluaciones del desarrollo a todos los niños del estudio. Los evaluadores independientes del equipo de intervención y ciegos a la asignación del tratamiento completaron todas estas medidas. Antes de que comenzara el estudio, la escala de observación de diagnóstico de autismo, conocida como ADOS y así como una entrevista, conocida como ADI-R, se utilizaron para validar el diagnóstico clínico de autismo del niño. Además, las Escalas de Mullen de Aprendizaje Temprano se utilizaron para informar la capacidad cognitiva general de cada niño. A todos los niños también se les administraron las Escalas de Desarrollo del Lenguaje de Reynell para evaluar las habilidades del lenguaje receptivo y expresivo.

También se aplicó la Escala de comunicación social temprana (ESCS). Esta escala es una medida de observación estructurada de 20 minutos que evalúa la aparición de habilidades de comunicación no verbal en niños pequeños. Esta evaluación se ha utilizado ampliamente con poblaciones con retraso en el 
desarrollo. Durante su puesta en práctica, un experimentador le presentó a un niño aproximadamente 20 situaciones diseñadas para ocasionar conducta no verbal con el propósito de atención conjunta, interacción social o regulación de la conducta. Éste se grabó en video y luego se puntuó. Un acto de atención conjunta se puso en práctica cuando el niño se comunicaba sobre un objeto, evento o persona con el propósito de compartir interés. La atención conjunta se puede mostrar a través de un gesto no verbal de señalar, coordinar la mirada, dar o mostrar un objeto. El afecto positivo compartido durante la atención conjunta se definió como cualquier caso de atención conjunta en la que el niño dirigía una sonrisa hacia un adulto. El afecto positivo compartido mediante enunciados durante la atención conjunta se definió como cualquier acto de atención conjunta que incluía una sonrisa dirigida hacia un adulto así como un enunciado verbal.

Tras completar el consentimiento informado y las evaluaciones cada niño fue asignado al azar a uno de las tres condiciones experimentales. Las sesiones del tratamiento se realizaron todos los días durante cinco o seis semanas. El propósito principal de la intervención de atención conjunta radica en incrementar la atención conjunta del niño durante las rutinas de juego desarrolladas especialmente mediante intervenciones de acercamiento. El objetivo de la intervención del juego simbólico estuvo en aumentar el nivel del niño y la frecuencia de los actos de juego según una escala de juego adaptada de Lifter et al. (1993). Por último, el propósito del grupo de control era representar el método estándar de tratamiento (aplicado al análisis del comportamiento) para niños con autismo.

Se utilizaron modelos de regresión de efectos mixtos para evaluar cambios en los siguientes tipos de calidad de la atención conjunta en cuatro momentos del estudio: afecto positivo compartido durante la articulación atención, así como afecto positivo compartido con la expresión durante la atención conjunta.

Examinamos el afecto positivo compartido durante la atención conjunta. Hubo una interacción para el grupo de atención conjunta así como para el grupo de juego simbólico. Las pruebas Z de seguimiento revelaron que los grupos de atención conjunta y juego simbólico no eran significativamente diferentes en el punto de partida, en el seguimiento de 6 meses o en el seguimiento de 12 meses.

También investigamos si la frecuencia del afecto positivo compartido con expresiones durante la atención conjunta aumentó dentro del grupo de tratamiento. Existía una interacción entre el grupo de atención conjunta, así como el grupo de juego simbólico. Las pruebas $\mathrm{Z}$ de seguimiento revelaron que los grupos de atención conjunta y juego simbólico no eran significativamente diferentes en el punto de partida, en el seguimiento de 6 meses o en el seguimiento de 12 meses.

Este estudio sugiere que cualquier tipo de intervención puede mejorar tanto la cantidad como la calidad de la atención conjunta. Además, sugiere que los niños en los grupos de atención conjunta y juego simbólico no sólo aprendieron cómo producir comportamientos específicos de atención conjunto, sino también que ambos grupos es muy probable que entendieron el verdadero propósito de la comunicación. 
Algunas de las hipótesis han sido refutadas mientras que otras no. Como era esperado algunos aspectos de la calidad de atención conjunta mejoraron en los niños con autismo, sin embargo no previmos que los niños que llevaron a cabo el juego simbólico también mostrarían mejoras en la calidad de la atención conjunta. También fue una sorpresa comprobar que no hubo una mejora significativa hasta las visitas de seguimiento. Esto se pudo haber debido a dos causas: primero, que en los niños con autismo puede ser más difícil mejorar la calidad de la atención conjunta más que la cantidad. La segunda razón es que el afecto positivo es posible que se mostrará más en ciertas habilidades de atención conjunta. Por lo tanto, se puede llegar a la conclusión de que futuras investigaciones deberían medir tanto la cantidad como la calidad de la atención conjunta, así como abordar las limitaciones de este estudio.

Por lo tanto, este estudio sugiere que cualquiera de las dos intervenciones puede mejorar tanto la cantidad como la calidad de la atención conjunta, las cuales están deterioradas en los niños pequeños con autismo. Si bien, desafortunadamente, en este momento no se puede saber con exactitud cómo la calidad de la atención conjunta podría variar como resultado de estas intervenciones.

“Clinical outcomes of interactive, intensive and individual (3i) play therapy for children with ASD: a two-year follow-up study" Pittala, Saint-Georges-Chaumet, Favrot, Tanet, Cohen, and Saint-Georges (2018)

El objetivo de este estudio es acercar a los niños con TEA a través del juego interactivo, individual e intensivo a la integración escolar ordinaria.

En lo que respecta a los participantes, los criterios de inclusión fueron los siguientes: estar diagnosticado con TEA según la Entrevista de Diagnóstico de Autismo Revisada (ADI-R) y la Escala de Calificación del Autismo Infantil (CARS); haber iniciado el método 3i entre Enero de 2013 y diciembre de 2013; residir en un ambiente que permita la correcta aplicación del método 3i; no recibir ningún otro método de tratamiento de intervención de TEA y que el francés sea su lengua materna.

Durante el período de selección, 31 niños comenzaron el programa 3i, pero solo 20 fueron incluidos en este estudio. Todos los sujetos incluidos no estaban asistiendo a ninguna escuela y seguían el método $3 \mathrm{i}$ entre 30 y 35 h por semana. La edad de los pacientes al inicio del estudio osciló entre 2,8 y 14,5 años.

El método 3i (individual, interactivo e intensivo) es promovido por AEVE, una asociación sin ánimo de lucro fundada en 2006. Se recomienda que este método sea intensivo: un mínimo de 30 h de sesiones de juego por semana en una sala de juego específica. Cada sesión de juego dura 1 hora y media. El tamaño recomendado de la sala de juegos es aproximadamente 10 metros cuadrados, la iluminación debe ser tenue y los sonidos deben amortiguarse con un revestimiento de suelo adecuado. El equipamiento estándar de la habitación incluye estanterías al alcance del niño, donde los objetos serán almacenados 
visiblemente, un espejo, un columpio y algunos otros elementos que se utilizan para obtener una percepción física de uno mismo. Las sesiones son dirigidas por voluntarios. La mayoría de estos participantes no son profesionales en el campo del TEA. Primero, los voluntarios son evaluados por los padres y después participan en una reunión con el director de AEVE, quien les proporciona información sobre el autismo y el método 3i. Dichos voluntarios son examinados en función de su capacidad para garantizar el cuidado de los niños antes de comenzar a interactuar con el niño en la sala de juegos. Posteriormente, participan cada mes en una sesión de grupo con los otros participantes, juntos con los padres, bajo la supervisión de un psicólogo entrenado en el método 3i. Este psicólogo capacitado en $3 \mathrm{i}$ dirige el equipo de voluntarios y asegura la coherencia de sus acciones y su cumplimiento con el método 3i. El progreso del niño es registrado y analizado por el psicólogo.

La intervención 3i se organiza en tres fases, que corresponden a los tramos de edad de desarrollo de 0 a 18 meses (Fase 1), 18 a 36 meses (Fase 2) y más 36 meses (fase 3). Durante la fase 1, la intervención se centra en simples juegos e intercambios sensoriales, sin usar muchos objetos. El objetivo es ayudar a los niños a descubrirse a sí mismos, su cuerpo y la existencia de otra persona. Durante la fase 2, la atención del niño se centra en el mundo exterior fuera de la sala de juegos, emerge un lenguaje significativo y el niño obtiene acceso al juego simbólico. El deseo del niño de un aprendizaje consciente aparece en la fase 3 y conduce a la integración progresiva en un aula como compañero de clase, sin ser visto como un niño discapacitado.

Se utilizaron diferentes medidas para evaluar el grado de autismo de cada uno de estos niños. En primer lugar, usamos VABS (Escalas de Comportamiento Adaptativo) para evaluar el impacto del método $3 i$ en el desarrollo de la socialización, la comunicación y la autonomía en su vida diaria. Por otro lado, también se utilizó NIS. El objetivo de esta medida era incluir también niños con discapacidad intelectual severa. Éste contiene tres elementos: imitación espontánea, reconocimiento de emulación e imitación por petición. En tercer lugar se usó la prueba PEP-R para calcular la edad de desarrollo del paciente en diferentes momentos de evaluación en las siguientes áreas de desarrollo: imitación, percepción, motricidad fina, habilidades motoras globales, coordinación óculo-manual, rendimiento cognitivo y cognición verbal de acuerdo con el comportamiento observado. En este estudio en concreto, esta prueba se centró en la imitación y la cognición verbal de la edad de desarrollo del niño, puesto que se consideraron aquellos requisitos previos más importantes para el (re)ingreso exitoso en la escuela. ADI-R es una herramienta de evaluación de diagnóstico basada en la descripción e historia del paciente que analiza su desarrollo en tres áreas: calidad de las interacciones sociales, comunicación y lenguaje, e intereses restringidos y comportamientos estereotipados. Este método se utilizó 3 veces durante este estudio como 
criterio secundario para evaluar la eficacia del método. Finalmente, CARS fue utilizado en este estudio para caracterizar la intensidad del trastorno autista y su evolución durante el tratamiento.

Todos los participantes fueron diagnosticados con TEA, de los cuales el 90\% eran niños (18 de 20). La edad cronológica en el punto de partida varió entre 33 y 173 meses. Además, el 65\% de los sujetos fueron categorizados inicialmente como no verbales con respecto a su modo de comunicación. En general, los sujetos pasaron $2832 \pm 550 \mathrm{~h}$ siguiendo el método $3 \mathrm{i}$ durante dos años, una media de $4 \mathrm{~h}$ de sesiones de $3 \mathrm{i}$ al día. Los veinte sujetos completaron las evaluaciones de inicio y las del primer año, sin embargo, tres sujetos abandonaron después de un año.

Una vez analizados los resultados, podemos observar que el desarrollo de la edad de la comunicación según VABS aumentó significativamente. Además, la edad de desarrollo de socialización según VABS aumentó significativamente representando un aumento del 83\% después de 2 años del método 3i. La puntuación NIS aumentó significativamente en un 53\%, lo que es consistente con el aumento del $67 \%$ de la imitación de la edad de desarrollo puntuada por PEP-R. La puntuación de cognición verbal PEP-R aumentó solo en un 23\% después 24 meses, lo que no fue significativo. Asimismo, de los otros 8 elementos evaluados por VABS y PEP-R, todos ellos aumentaron significativamente durante los 24 meses del estudio, excepto las habilidades motoras globales de PEP-R. Los resultados generales sugieren que 24 meses del método $3 \mathrm{i}$ se asocia a un aumento de las habilidades de desarrollo y comportamiento entre los 20 sujetos.

En lo que respecta al nivel de autismo de los niños, el $94 \%$ de nuestros pacientes fueron diagnosticados con un autismo severo al comienzo del estudio y el $6 \%$ de ellos con un nivel moderado de autismo. Al final de los 2 años, solo el 21\% (4/19) permaneció gravemente autista, el 53\% (10/19) había progresado a un autismo moderado, y 26\% (5/19) podría considerarse que ya no tiene autismo. Si bien, todos los individuos todavía tenían una discapacidad intelectual de moderada a severa al final del estudio.

Curiosamente, el cambio en la puntuación de socialización de VABS fue correlacionado positivamente con la duración total de la recepción el método 3i. Esta variable fue el único resultado principal que continuó aumentando significativamente en T2 en comparación con T1. Por el contrario, no se encontró correlación entre la edad de los sujetos y la disminución general de la gravedad de TEA o su progreso en las habilidades de desarrollo.

Como conclusión se puede afirmar que este es el primer estudio prospectivo que sugiere los efectos positivos del método $3 \mathrm{i}$ sobre las habilidades de comportamiento y desarrollo así como la gravedad del TEA. Los resultados presentados en este estudio están en línea con estudios retrospectivos previos sobre el Intervención 3i. Sin embargo, es necesario realizar más estudios para respaldar esos resultados iniciales. 


\section{DISCUSIÓN DE LOS RESULTADOS}

Los artículos escogidos han sido divididos en dos grupos: el primer grupo incluye aquellos artículos relacionados con la terapia parental, mientras que el segundo grupo se centra en la terapia de juego.

En lo que respecta al primer grupo, encontramos dos artículos. El primero de ellos, denominado "The Effect of Filial Therapy on the Parenting Stress of Mothers of Children With Autism Spectrum Disorder", presenta unos resultados positivos. Asimismo, también ha reforzado la unión padre-hijo, gracias a la reducción de dicho estrés por parte de los padres.

El segundo artículo relacionado con la terapia parental, "Effects of a Brief Early Start Denver Model (ESDM) - Based Parent Intervention on Toddlers at Risk for Autism Spectrum Disorders: A Randomized Controlled Trial”, presenta unos resultados completamente distintos al artículo anterior. Este trabajo no fue diseñado correctamente desde su inicio, puesto que el grupo de control recibió más horas de intervención que el grupo que llevó a cabo el modelo P-ESDM. Por tanto, no existen diferencias significativas entre ambos grupos.

Debido al bajo número de muestras en lo que se refiere a los artículos relacionados con la terapia parental, no podemos sacar unas conclusiones significativas. Tal y como podemos observar, uno de estos estudios ha sido exitoso, mientras que el otro no ha alcanzado las metas planteadas. Sin embargo, consideramos que de haberse diseñado correctamente la segunda de estas investigaciones, podría haber tenido un resultado positivo. Por ello, y en relación con nuestro objetivo sobre si la intervención de los padres influye de manera positiva en los niños con TEA, podemos sugerir que este tipo de intervenciones sí ayuda realmente en las intervenciones de los niños con TEA, puesto que al reducir el estrés parental, mejora notablemente la relación padre-hijo, y por tanto la socialización de estos niños con sus padres y/o entorno más cercano. Sin embargo, debido al bajo número de muestras, no podemos respaldar nuestro objetivo completamente.

Por otro lado, encontramos cuatro artículos relacionados con la terapia de juego. El primero de estos artículos se llama “Using Augmented Reality to Elicit Pretend Play for Children with Autism”. Se ha observado que efectivamente, dicho modelo de realidad aumentada ha aumentado el tiempo de juego simbólico en dichos niños, especialmente en aquellos con un mayor retraso en el desarrollo. Sin embargo, el reto está en llevar estos resultados a la vida real.

El segundo artículo, "Cognitive rehabilitation system for children with autism spectrum disorder using serious games: A pilot study”, Tras dicha intervención, se deduce que este sistema podría ayudar a la rehabilitación cognitiva y trabajar el área de la interacción afectada, si bien existen ciertas limitaciones, 
pues los niños trabajan con la aplicación pero es necesaria la intervención social. Concluyen también que las nuevas tecnologías abren la puerta a nuevas terapias de intervención.

El tercero de los artículos escogidos, "Brief Report: Longitudinal Improvements in the Quality of Joint Attention in Preschool Children with Autism", Tras llevar a cabo dichas terapias, se puede comprobar que, si bien aumentaron la calidad de la atención conjunta tanto en la terapia de afecto compartido como en el juego simbólico, no existe una diferencia significativa con el grupo de control. Por tanto, se puede concluir que es efectiva hasta cierto punto, si bien precisa de estudios posteriores.

El último artículo es el denominado "Clinical outcomes of interactive, intensive and individual (3i) play therapy for children with ASD: a two-year follow-up study. Este estudio difiere de los estudios anteriores en lo que respecta a los participantes, ya que tenían deficiencias cognitivas además de presentar síntomas de trastorno del espectro autista. Los resultados de este estudio son muy positivos, puesto que aumentó significativamente la edad de desarrollo de socialización, la imitación, la puntuación de cognición verbal, las habilidades de desarrollo y comportamiento, así como habilidades motoras. Además, la gravedad del autismo se redujo en la mayoría de los casos, considerándose que un $26 \%$ de los niños ya no tenía autismo.

Estos cuatro artículos muestran que, si bien las nuevas tecnologías abren la puerta a un gran abanico de posibilidades, es necesario continuar investigando nuevas formas de intervención. También se puede observar que la interacción social y emociones juegan un papel fundamental en el desarrollo de niños con TEA. Por lo tanto, y en lo que respecta a nuestro segundo objetivo, podemos afirmar que todos los autores examinados están de acuerdo en que la terapia de juego, así como las nuevas tecnologías, son un aliciente para socializar con niños con TEA. Sin embargo, contrastando los resultados obtenidos en estos artículos, podemos afirmar que si bien las nuevas tecnologías atraen la atención de los niños con trastorno del espectro autista, es necesario una mayor investigación que permita llevar esos aprendizajes a la práctica en el mundo real. Es por ello que consideramos que nuestro objetivo se ha cumplido parcialmente, pues si bien las terapias de juego y las nuevas tecnologías ayudan en las intervenciones, es necesario desarrollar en profundidad la forma en que llevar estos aprendizajes a la práctica en la vida diaria de estos niños.

Por otro lado, en relación a nuestro tercer objetivo, como bien hemos visto en los artículos seleccionados, todas estas técnicas alternativas ilustradas en estos artículos han sido llevadas a cabo en la actualidad, entre 2010 y 2020. Sin embargo, las hipótesis que se pretenden corroborar son muy novedosas, llevándose a la práctica en la mayoría de los casos por primera vez, y por tanto no podemos afirmar que se lleven a cabo en el día a día en las terapias de intervención. El único de ellos que realmente se ha 
llevado en algún momento a la práctica, y que realmente tiene resultados muy positivos, es el método $3 \mathrm{i}$. Sin embargo, no es método totalmente establecido y utilizado en los tratamientos actuales.

Por último, podemos afirmar que el objetivo principal de este artículo sí se ha cumplido con éxito. Se puede observar que en todos los artículos realmente hay una mejora significativa en el diagnóstico de los niños con TEA al llevar a cabo los diferentes métodos de intervención. Todos los niños mediante cualquier tipo de intervención ya sea el tratamiento habitual, juegos o la terapia parental mejoran respecto a su diagnóstico inicial del Trastorno del Espectro Autista. Sin embargo, en algunas ocasiones no hay unas diferencias significativas entre el grupo clínico y el grupo de control, ya que este último también lleva a cabo otras técnicas e intervenciones habituales. Es por esto que en algunas ocasiones las intervenciones que se llevan a cabo no mejoran de manera significativa en cuanto al grupo de control, pero sí existe una mejora como tal.

\section{CONCLUSIONES}

Nuestro objetivo principal en este estudio era comprobar si la terapia parental y la terapia de juego son beneficiosas en los tratamientos con niños que padecen Trastorno del Espectro Autista, así como verificar si dichos tratamientos se llevan en la práctica en la actualidad. Sin embargo, hemos encontrado algunas limitaciones durante la realización de este artículo. En primer lugar, el bajo número de muestras que hemos obtenido utilizando las palabras clave previamente mencionadas. Consideramos que el uso de otras palabras clave podría haber ayudado a encontrar un mayor número de artículos, y por tanto podríamos haber analizado en profundidad la temática seleccionada.

En segundo lugar, todos los artículos seleccionados estaban escritos en lengua inglesa. Si bien la mayoría de ellos se habían llevado a cabo en países de habla inglesa, uno de ellos se había llevado a cabo en Francia. Es por ello que no podemos afirmar si estas investigaciones se llevan a la práctica en nuestro país en la actualidad, sin embargo, resultaría muy interesante poner en práctica en España algunos de estos estudios. Asimismo, ninguno de ellos se lleva a la práctica en sus países de origen, a excepción del método 3i, el cual sí se había llevado a cabo anteriormente.

Por lo tanto, con este estudio se sugiere que la terapia parental y la terapia de juego son beneficiosas en niños con TEA en lo que respecta la intervención temprana. Sin embargo, sería necesario llevar a cabo una segunda revisión sistemática para poder corroborar nuestras impresiones con un mayor número de artículos, incluyendo también artículos realizados en España. Para ello, sugerimos que durante la búsqueda de artículo se incluyan otras palabras clave diferentes, así como otras bases de datos distintas que incluyan artículos en español y en inglés. 
Y es que, vivimos en una sociedad enriquecida con la originalidad de que cada persona es diferente y posee sus propias peculiaridades. El entorno que nos rodea es vital para que el desarrollo de cada uno de nosotros sea de una manera u otra. En lo que respecta a los niños, cada uno es completamente distinto, y por tanto, habrá ciertas terapias que sean efectivas con algunos niños, y otras terapias con otros. Tal y como dijo Eric Kandel (2007) "Como cada ser humano se cría en un ambiente diferente y también tiene experiencias distintas, la arquitectura de cada cerebro humano es única." 


\section{REFERENCIAS}

American Psychiatric Association. (2013). Diagnostic statistical manual of mental disorders. (5 ${ }^{\mathrm{a}}$ ed.). American Psychiatric Publising. file:///C:/Users/34652/Downloads/APA_DSM-5-Autism-SpectrumDisorder.pdf

Aresti-Bartolome, N., \& Garcia-Zapirain, B. (2015). Cognitive rehabilitation system for children with autism spectrum disorder using serious games: A pilot study. Bio-Medical Materials and Engineering, 26, 811-824.

Autismo Diario (2015). Fomento de las habilidades del niño con autismo a través del juego. Autismo Diario: Autismo Diario https://autismodiario.com/2015/04/05/fomento-de-las-habilidades-del-nino-conautismo-a-traves-del-juego/

Bai, Z., Blackwell, A., y Coulouris, G. (2015). Using Augmented Reality to Elicit Pretend Play for Children with Autism. IEEE Transactions on Visualization and Computer Graphics. 21(5). 598-610. doi: 10.1109/TVCG.2014.2385092

Confederación de autismo de España. (s.f.). Sobre el TEA. Detección y diagnóstico. Confederación de Autismo España: Confederación Autismo España $\underline{\text { TEA/deteccion }}$

Confederación de autismo de España. (s.f.). TEA: Trastorno del Espectro Autista. Detección y diagnóstico. Confederación de Autismo España: Confederación Autismo España http://www.autismo.org.es/sobre-los-TEA

Ebrahimi, E., Hosseinzadeh, S., Sourtiji, H., Hosseini1, A., Mirzai1.H., \& Kiyani, Z. (2020). Children of Mothers of Stress Parenting the on Therapy Filial of Effect The r Spectrum Autism Wit. Arvhives oh Rehabilitation, 21(2), 206-219.

García, A. (2008). Espectro Autista: definición, evaluación e intervención educativa. Mérida: Junta de Extremadura.

González Moreno, C.X. (2018). Intervención en un niño con autismo mediante el juego. Revista de la Facultad de Medicina, 66(3), 365-374.

Grupo de Atención Temprana (GAT) (2000). Libro Blanco de la Atención Temprana. Madrid: Ministerio de Trabajo y Servicios Sociales. Real Patronato de Prevención y de Atención a Personas con Minusvalía.

Hernández, J.M., Artigas-Pallarés, J., Martos-Pérez, J., Palacios-Antón, S., Fuentes-Biggi, J., BelinchónCarmona, M., Canal-Bedia, R., Díez-Cuervo, A., Ferrari-Arroyo, M.J., Hervás-Zúñiga, A., IdiazábalAlecha, M.A., Mulasl, F., Muñoz-Yunta, J.A., Tamarit, J., Valdizán, J.J., \& Posada-De la Paz, M. (2005). Guía de buena práctica para la detección temprana. Revista de Neurología, 41(4), 237-245.

Hervás Zúñiga, A., Balmañna, N., \& Salgado, M. (2017). Los trastornos del espectro autista (TEA). Pediatría Integral, XXI(2), 92-108.

Kandel, Eric R. (2007) En busca de la memoria: nacimiento de una nueva ciencia de la mente. Buenos Aires: Katz. 
Lawton, K., \& Kasari, C. (2011). Brief Report: Longitudinal Improvements in the Quality of Joint Attention in Preschool Children with Autism. Springer, 42, 307-312.

López Gómez, S., Rivas Torres, R.M., \& Taboada Ares, E.M. (2010). Historia del Trastorno Autista. Apuntes de psicología, 28(1), 51-64.

Martínez- González, R.A., \& Becedóniz Vázquez, C.M. (2009). Orientación Educativa para la Vida Familiar como Medida de Apoyo para el Desempeño de la Parentalidad Positiva. Psychosocial Intervention, 18(2), 97-112.

Moreno, E. (2010). Políticas de infancia y parentalidad positiva en el marco europeo. Papeles Salmantinos de Educación, 14, 17-28.

Muñoz Yunta, A., Palau, M., Salvadó, B., \& Valls, A. (2006). Autismo: identificación e intervención temprana. Acta Neurol Colomb, 22, 97-105.

Organización Mundial de la Salud. (2021). Trastorno del Espectro Autista. Organización Mundial de la Salud: Organización Mundial de la salud https://www.who.int/es/news-room/fact-sheets/detail/autismspectrum-disorders

Palomo, R. Arnáiz, J. Zamora, M. Márquez, C. y García, R. (2014). Documento de reflexión en torno a los cambios propuestos en el DSM-5 en relación con los Trastornos del Espectro del Autismo y su aplicación en España. Asociación Española de Profesiones del Autismo: Asociación Española de Profesiones del

Autismo http://horarioscentros.uned.es/archivos_publicos/qdocente_planes/1037974/dsm5reflexionaetapi2014.pd $\underline{f}$

Pittala, E.T., Saint-Georges-Chaumet, Y., Favrot, C., Tanet, A., Cohen, D., \& Saint-Georges, C. (2018). Clinical outcomes of interactive, intensive and individual (3i) play therapy for children. BMC Pediatrics, 18(165), 1-13.

Rodríguez-Barrionuevo, A.C. \& Rodríguez-Vives, M.A. (2002). Diagnóstico clínico del autismo. REV NEUROL, 34(1), 72-77.

Rogers, S.J., Estes, A., Lord, C., Vismara, L., Winter, J., Fitzpatrick, A., Guo,M., \& Dawson, G. (2012). Effects of a Brief Early Start Denver Model (ESDM) - Based Parent Intervention on Toddlers at Risk for Autism Spectrum Disorders: A Randomized Controlled Trial. New Research, 51(10), 1052-1065.

Schaefer, C. (2012). Fundamentos de Terapia de Juego. México: El manual moderno.

Torres López, D. (05 de marzo de 2015). El juego pintado de azul: desarrollo de la comunicación social y el lenguaje en niños con Trastorno del Espectro del Autismo (TEA) por medio de la participación guiada en actividades lúdicas. Recuperado de ttp://www.difver.gob.mx/cedaa/wpcontent/uploads/sites/6/2015/07/ART\%C3\%8DCULO_Juego_Autismo_David_Torres_M\%C3\%89XIC $\underline{\text { O.pdf }}$

Wing, L., y Gould, J. (1979). Severe impairments of social interaction and associated abnormalities in children: Epidemiology and clasification. Journal of Autism and Developmental Disorders, 13, 255-267. 
